\title{
High Incidence Of Fever In Patients After BioIntegral Pulmonic Valved Conduit Implantation
}

\author{
Arif Selcuk ${ }^{1}$, Yiğit Kılıç ${ }^{1}$, Oktay Korun ${ }^{1}$, Okan Yurdakök ${ }^{1}$, Murat Çiçek ${ }^{2}$, Firat Altin ${ }^{3}$, \\ Yasemin Altuntas ${ }^{2}$, Emine Hekim Yilmaz ${ }^{1}$, Ahmet Sasmazel ${ }^{4}$, and Numan Aydemir ${ }^{5}$ \\ ${ }^{1}$ Dr Siyami Ersek Thoracic and Cardiovascular Surgery Training and Research Hospital \\ ${ }^{2}$ Doktor Siyami Ersek Gogus Kalp ve Damar Cerrahisi Egitim ve Arastirma Hastanesi \\ ${ }^{3}$ ISTANBUL MEHMET AKIF ERSOY THORACIC AND CARDIOVASCULAR \\ SURGERY TRAINING AND RESEARCH HOSPITAL \\ ${ }^{4}$ Kartal Kosuyolu Heart Education and Research Hospital \\ ${ }^{5}$ Dr.Siyami Ersek THORACIC AND CARDIOVASCULAR SURGERY TRAINING AND \\ RESEARCH HOSPITAL
}

February 19, 2021

\begin{abstract}
OBJECTIVE The aim of this study is to describe our short-term outcomes using BioIntegral pulmonic conduit. METHODS Between August 2018 and September 2019, the BioIntegral pulmonic valved conduit was used for right ventricular outflow tract reconstruction in 48 patients. The data was retrospectively retrieved from the patient charts. RESULTS The median age at the operation was 36 months (IQR:18-62 months). The diagnoses were PA-VSD in 28 patients, absent pulmonary valve in four patients, truncus arteriosus in six patients, TGA-VSD-PS in five patients, conduit stenosis in three patients and LVOT obstruction requiring Ross operation in two patients. In the postoperative follow-up 15 patients out of 48 had high fever. Out of these, 12 patients had concomitantly elevated CRP levels. There were no patients with blood culture positivity. The median postoperative length of hospital stay was 14 days (IQR:8-21 days). The overall mortality was recorded in 2 patients (4\%), one died due to right ventricular failure and multiple organ failure and one died due to pulmonary embolism. The two patients who died were not among the 15 patients with fever. CONCLUSIONS There was high incidence of fever and adverse outcomes in the short-term postoperative follow-up of the patients in whom the BioIntegral pulmonic valved conduit was implanted. Caution is advisable in using these conduits until there is convincing evidence about the sterilisation and storage standards of these grafts.
\end{abstract}

\section{Hosted file}

text.doc available at https://authorea.com/users/356812/articles/509785-high-incidence-offever-in-patients-after-biointegral-pulmonic-valved-conduit-implantation 


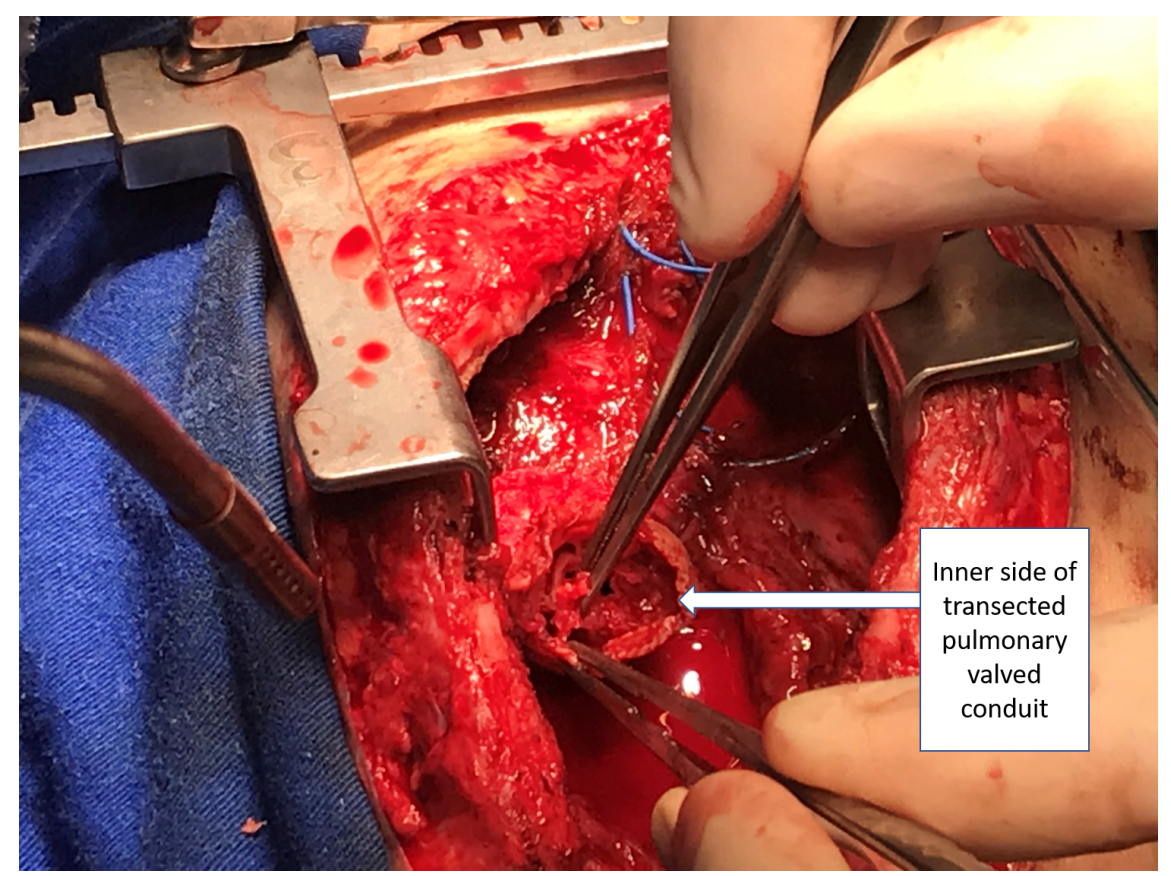

